\title{
Measuring the scattering mean free path in heterogeneous media
}

\author{
Anne Obermann, Éric Larose, Vincent Rossetto, Ludovic Margerin
}

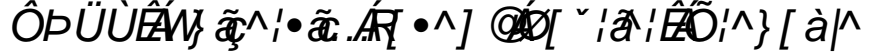




\section{Measuring the scattering mean free path in heterogeneous media}

\section{Anne Obermann, Éric Larose, Vincent Rossetto, Ludovic Margerin}

Goal:

Study the phase statistics in coda waves and determine the scattering mean free path $\ell$ from spatial phase decoherence.

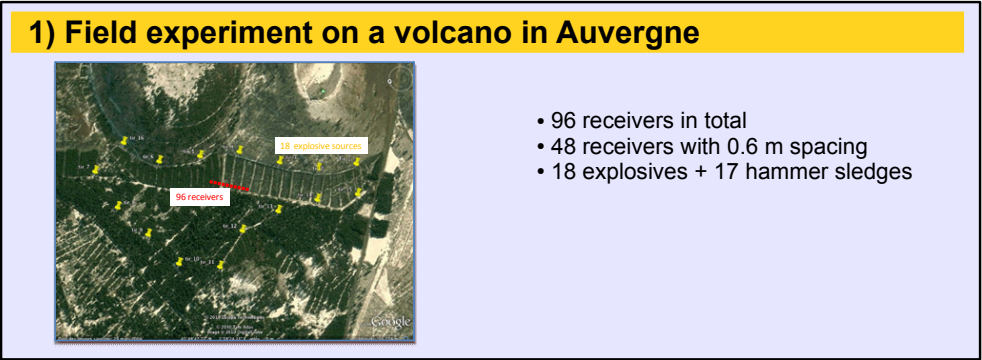

\section{2) Raw data + Processing steps}
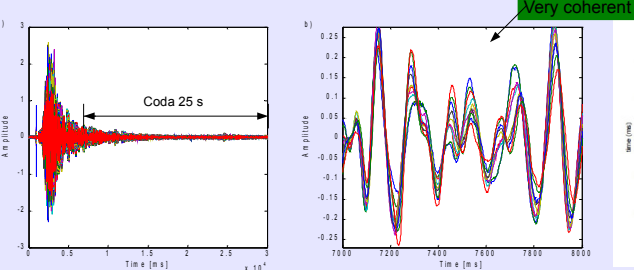

Processing steps

- Bandpassfilter: $30 \mathrm{~Hz} \pm 5 \%$

- Hilbert transformation - Select time window - Spatial phase unwrapping

\section{3) Gaussian statistics}
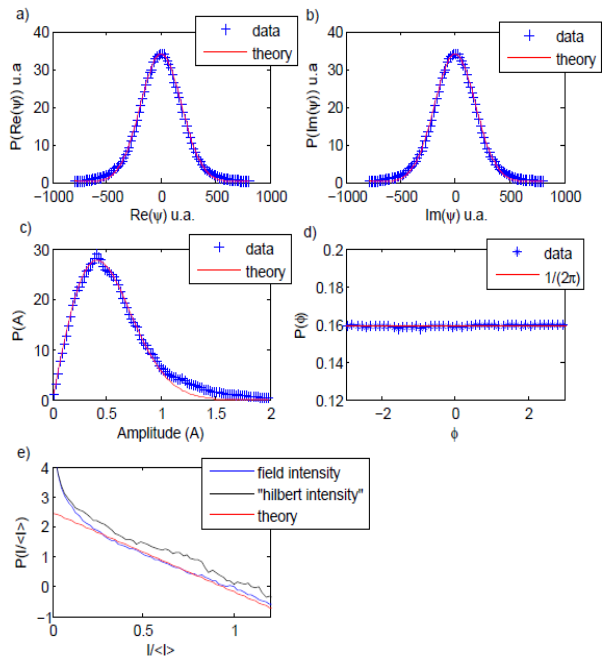

Criteria for signals that obey circular gaussian statistics:

a) + b) The real and imaginary part of the field follow a Gaussian distribution

c) The amplitude distribution follows a Rayleigh distribution

d) The probability distribution of the phase is uniform

e) The amplitude decays exponentially

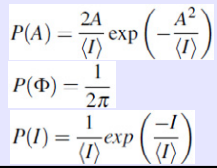

\section{4) Field correlation}

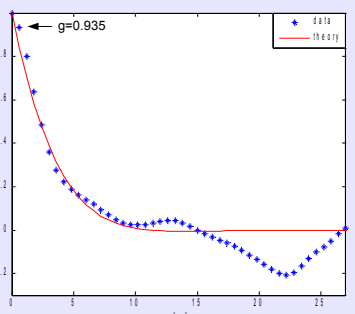

$$
C(r)=J_{0}(k r) \exp (-\stackrel{r}{2 l})
$$

\section{5) Phase difference distributions}

First phase derivative distribution

Wrapped phase $\Phi$

Unwrapped phase $\Phi_{u}$
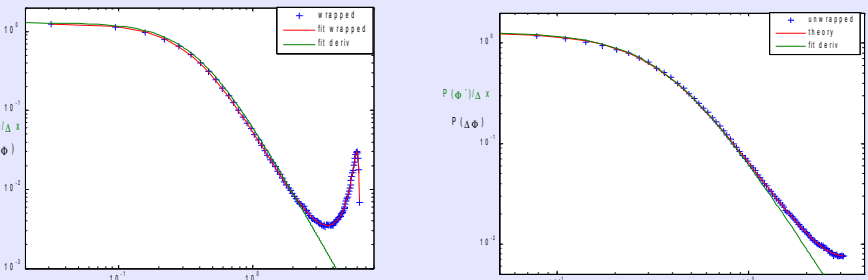

$P(\Delta \Phi)=\frac{2 \pi-|\Delta \Phi|}{4 \pi^{2}}\left[\frac{1-g^{2}}{1-F^{2}}\right]\left[1+\frac{F \cos ^{-1}(-F)}{\sqrt{1-F^{2}}}\right]$
$F=g_{1} \cos (\Delta \Phi)$

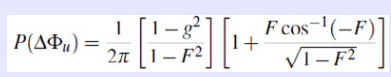

$F=g_{1} \cos \left(\Delta \Phi_{u}\right)$

$$
P\left(\Phi^{\prime}\right)-\frac{1}{2} \frac{Q}{\left[Q+\Phi^{\prime 2}\right]^{3 / 2}} \Rightarrow Q=-C^{\prime \prime}(0) \simeq \frac{1}{2} k^{2}
$$

Higher order phase derivative distributions
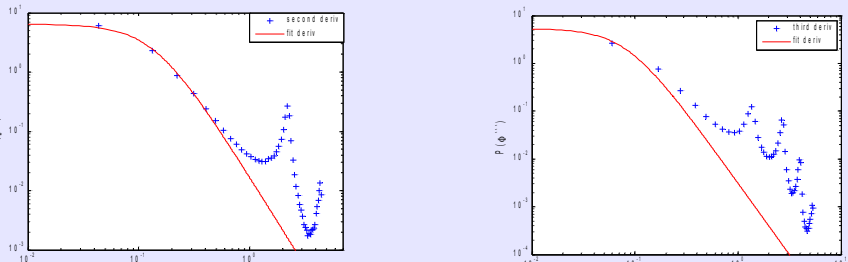

\section{$Q=0.155$}

6) Phase difference correlation
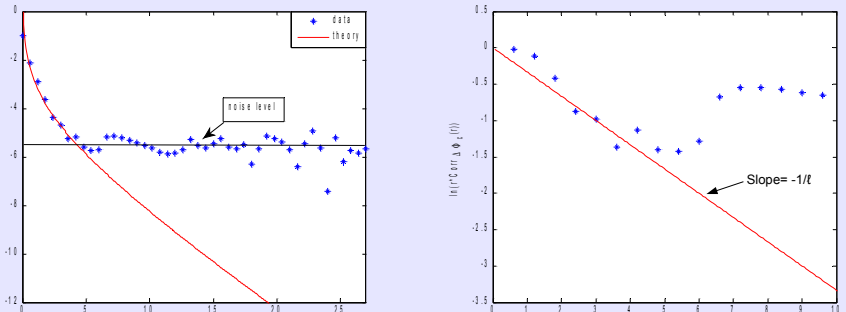

$C_{\Phi^{\prime}}(r>\lambda) \longrightarrow \frac{1}{2}\left[\left(C^{\prime}\right)^{2}-C^{\prime \prime} C\right]=(k / \pi r) \exp (-r / l)$

Comparison to numerical simulation:

48 receivers

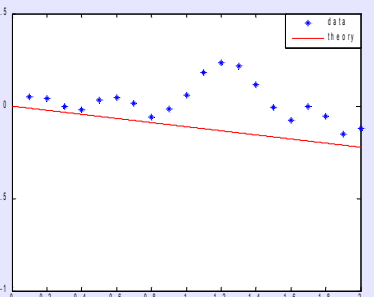

\section{7 receivers}

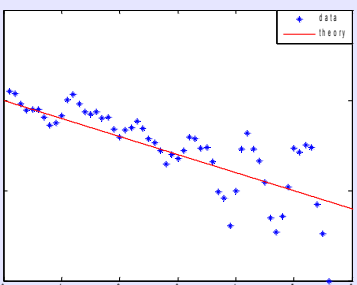

